\title{
Lived Experiences of Emergency Department (ED) Nurses with Comorbidities amidst COVID-19 Pandemic at Candon City of Ilocos Sur: A Phenomenological Study
}

\author{
Mariecris Talosig ${ }^{1}$, Romeo Sanchez $\mathrm{II}^{2}$, Gevin Soriano ${ }^{3}$ \\ ${ }^{1,2,3}$ St. Paul University Philippines \\ Corresponding Author: Mariecris Talosig
}

\begin{abstract}
Nurses may be regarded as modern-day superheroes, but realistically, they are humans too. They experience joy, gratitude, fear, and anxiety just like normal human beings. With this pandemic, nurses are exposed to psychological stress. Healthcare staff, specifically nurses, experience a plethora of psychological distress during the care of COVID-19 patients. Identifying factors that affect nurses' mental health during the care of these patients can be an advantage in mitigating the psychological burden that they are experiencing. ${ }^{[1]}$ This study described the ER nurses' lived experiences about being in the frontline despite their comorbidities. The study applied a qualitative method using Husserlian phenomenology. The researchers adopted the purposive sampling approach in selecting the ten participants to achieved data saturation. The process involved in the collection of data was through individual interviews. The criteria of the selection of participants are: must be registered nurse, a nurse working at the emergency room of the four hospitals in Candon City, Ilocos Sur; with a minimum work experience of 6 months in the facility; between ages 40 to 60 years of age; regular employee; and with comorbidity.
\end{abstract}

Keywords: lived-experiences, emergency room nurses, comorbidity, amid pandemic

\section{INTRODUCTION}

The Coronavirus Disease 2019 (COVID-19) pandemic has had a profound impact worldwide. It created an enormous shock for governments, administrative agencies, healthcare sectors, businesses, and ordinary people. As well as the immediate threat to public health, the pandemic is producing substantial economic and social stresses as societies introduce measures to restrict the proliferation of the virus. According to World Health Organization (WHO), a pandemic occurs when a disease proliferates internationally, traverses international borders, and affects many people. A pandemic can be said to transpire yearly in each of the temperate hemispheres, given that cyclical epidemics pass over foreign borders and impact a vast number of individuals. [2]

The pandemic crisis that the world has been facing since 2019 is COVID-19. COVID-19 was initially recorded in Wuhan, China, in 2019, and accordingly, originated the novel severe acute respiratory syndrome (SARS-Cov-2). [3]

In 2021, the International Council for Nurses stated that the ubiquity of COVID-19 had influenced the community locally and worldwide, especially those working in healthcare institutions who are nurses. Nurses are considered the gems of healthcare institutions. As the world battle against COVID-19, nurses are working 
diligently to render services to the sick and address health concern to the community. Nursing is bound to independent and collaborative care of people from all generations, ancestry, class, locality, ill or well, and all settings. Nursing incorporates the advancement of health, prevention of illness, and caring for sick, incapable, and dying individuals. In addition, advocacy, development of a safe setting, research, forming health guidelines and inpatient and health systems administration, and education are also key nursing roles.

The preponderance of affected people seeks medical treatment in medical facilities, particularly hospitals; a multitude of cases, especially those critically ill, will have repercussions on hospitals. [4] Nurses are on the frontline and are in charge of ensuring that all types of patients receive holistic care. Nurses have a critical function in healthcare systems because they are the predominant healthcare providers. [5] They are responsible for providing medical assistance and care for patients with COVID-19, screening patients, and recognizing suspected cases with infections. They also contribute to the delivery of necessary treatment and are involved in precautious dealing with suspected patients, assist in the process of decontamination and coordinate with other healthcare providers. In addition, nurses supply holistic practices in managing multiple infections simultaneously, play a critical role in expanding care services, and deal with relatives. [6] Emergency Department (ED) nurses are one of the front-liners who are currently battling this pandemic for more than a year now.

The importance of exploring the concerns that ED nurses withstanding Covid-19 patients will help increase the resilience of nurses and the hospital, in general, on how to deal with the crisis, strengthen readiness and accelerate recovery from the situation. [7]

Furthermore, awareness of issues concerning nurses helps nursing leaders and hospital administrators in creating decisions and recommendations. Therefore, this study aims to explore the lived experiences of ED nurses with comorbidities in their response to the crisis we all know as COVID-19.

\section{The Problem or Research Question}

Following the discussion, this research work is intended to describe the lived experiences of COVID-19 nurse frontliners who have comorbidities engaged in the Ilocos Region with the following questions:

Research Question 1. What are your thoughts in the face of this pandemic?

Research Question 2. How did you feel when accepting the anti-epidemic task?

Research Question 3. How do you feel when working in such a high-risk workplace?

Research Question 4. How do you take care of your personal and family needs while working?

Research Question 5. What are your coping strategies?

\section{Philosophical Underpinnings}

Due to enormous nursing function, lengthy work hours, shift duties, and working in a fast-paced and high-risk environment, Emergency Department nurses are constantly faced with immense psychological pressure. [8,9] Fatigue, burnout, mental exhaustion, and emotional detachment are the predicaments the nurses are dealing with in physically and emotionally challenging situations. [10] COVID-19 pandemic brought anxiety and depressive symptoms, emotional breakdown, and sleep disturbance to frontline clinicians, including nurses, especially those who have close contact with affected patients. Also, they regularly experience these negative emotions and symptoms due to the limited clinical understanding of the novel coronavirus and low protective gear and other medical supplies. [11, 12] Moreover, fear of contracting the virus prevails because Covid-19 is unfamiliar and indiscernible to the naked eye. However, frontline workers' sentiments, worries, perceptions, or 
thoughts often dwindle due to virtues which many frontline workers live, such as altruism and heroism. The call to serve remains amid this worldwide pandemic.

The Theory of Comfort is used as a practical guide in exploring the lived experiences of ED nurses that have comorbidities in handling COVID-19 patients in Ilocos Sur.

Theory of Comfort was coined by an American middle-range nurse theorist Katharine Kolcaba in 1994. This theory is anchored on these three concepts that include relief, ease, and transcendence; and goes around into four domains that include physical, psychospiritual, sociocultural, and environmental. Relief is defined as the experience of having comfort needs that have been met. Meanwhile, ease is defined as the experience of care that stimulates calmness and contentment. And transcendence is the experience in which care allows an individual to rise beyond predicament or pain. [13]

The physical domain of the theory of comfort regards all physiological and homeostatic dimensions of a person. The psychospiritual part goes into the internal understanding of esteem, self-concept, sexuality, life meaning, and relation to a higher power. The sociocultural domain of the theory mentioned above encompasses interpersonal, family, and societal connections, including finances, teaching, healthcare personnel, family traditions, rituals, and religious practices. The environmental domain talks about the external background of human experience such as temperature, light, sound, odor, color, furniture, landscape, and others that a nurse or loved one can control to boost comfort. [13]

The theory, as mentioned above, accounts for patients as individuals, families, institutions, or communities needing health care. The concept of health is the optimal functioning in the patient, as defined by the patient, group, family, or community. In this research, ED nurses with comorbidities handling COVID-19 clients are identified as patients in dire need of holistic health care to achieve optimal functioning.

Nurses deserve the same comfort that they provide to their patients. They also deserver the care of a professional environment in which to work. They must find ways to nurture themselves even in undesirable conditions to help them become resilient in response to the crisis.

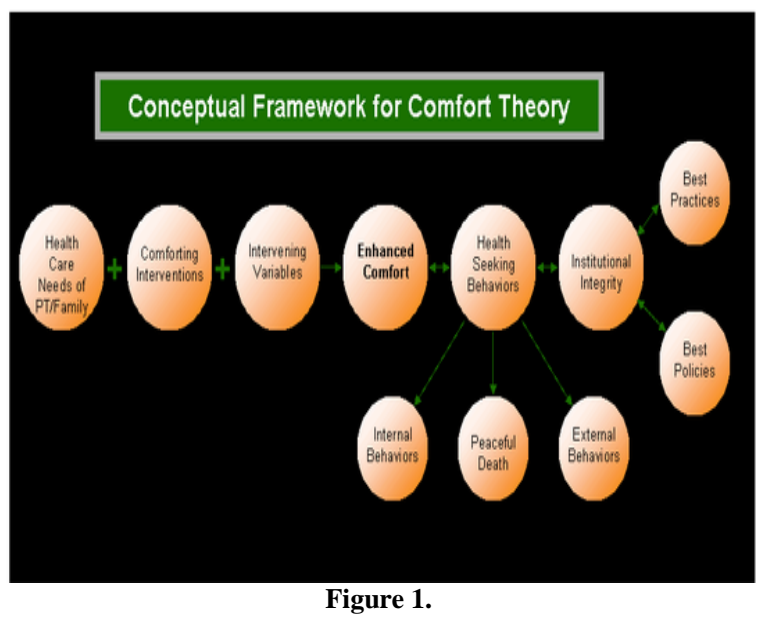

\section{METHODOLOGY}

This section presents the processes and procedures that the researchers used in the conduct of this study. It includes the design used in creating this research work, participants selected for this study, instrumentation, data collection procedure, analysis of data, and preliminary biases.

\section{Research Design}

The research study applied a qualitative approach using Husserlian phenomenology. Husserlian phenomenology is commonly used in social science research to explore and describe the lived experiences of individuals. Phenomenology is regarded as a wellfounded qualitative methodology that is frequently used and considered valuable in addressing nursing practice and education. [14] Through this, the researchers were able to gather information based on what happened in a particular phenomenon. These lived experiences are dimensions of being that had to be discovered and 
described through this study. The researchers intended to illustrate the structure of nurses with comorbidities experiences working in the emergency room with the threat of COVID-19 and to express their natural attitudes and thoughts towards the phenomenon.

The qualitative research method was chosen for this study. It appropriately shows the research's intent that helps us directly describe everyday lived experiences and unveil the findings using the respondent's own words and expressions. Employing the Husserlian phenomenology as an approach in this study creates a deeper understanding of their words and actions without biases, and it lessens any form of misunderstanding.

\section{Participants / Key Informants}

A non-probability sampling technique was utilized in this study, specifically purposive sampling. This technique allows the samples to be selected based on the characteristics of a population and the study's objective.[15] The researchers have determined 10 participants with the following inclusion criteria: (1) must be registered nurse, (2) nurse working at the emergency room of the four hospitals in Candon City, Ilocos Sur, (3) with a minimum work experience of 6 months in the facility, (4) between ages 40 to 60 years of age, (5) regular employee, and (6) with comorbidity. The researchers were limited to 3 days of data gathering. Furthermore, the researchers gathered data from the participants until data saturation was achieved. The schedule of interviews was conducted based on both the availability of the researchers and the participants. More importantly, the WHO Informed Consent was utilized to protect the rights of participants of this study. In the selection of participants, the researchers considered those nurses that are working in the emergency room. These are nurses that are highly exposed to different work-related risks, including Covid-19. A minimum work experience of 6 months indicates that the nurse has at least some form of familiarity, including the risk involved in working in such a high-risk department as the ED. Inclusion of nurses ages 40-60 as lifestylerelated diseases is expected in this age bracket. Hypertension associated with age is a significant risk factor in developing diseases involving the kidneys, cardiovascular system, stroke, and diabetes mellitus type 2. [16]. The inclusion of being a regular employee as a criterion eliminates the compensation factor in this study. A semi-structured interview and observations were used to gather data from nurses working in four hospitals in Candon City, Ilocos Sur. In utilizing the Husserlian approach, the researchers reserved their presuppositions, assumptions, and biases to attain accurate descriptions of participant's thoughts and feelings, being a mere frontline in the campaign against COVID19. An interview guide was utilized to promote a spontaneous and consistent conversation between the participant and the researcher. Moreover, appointments were scheduled for the interview for those participants who expressed their desire to participate in this research.

To gain the participant's cooperation, the nature and the purpose of this research work were discussed. Researchers established a trusting environment by showing respect, attentively listening to their responses, and asserting clarification to their answers. A recorded interview was employed with consent from the participants, who allowed the researchers to concentrate on the interview process. The researchers have listened to the recording then the transcription was made.

\section{Instrumentation}

An interview guide is a powerful instrument that was used in the study. It is semi-structured. The questions were flexible enough to ensure that they would fit the participant's feelings or reactions. An audio recording was also utilized during the interview. In this way, the researchers focused on the participants' responses while 
documenting their verbal reactions without taking notes. Also, this made it easier for the researchers to review participants' responses for better interpretation of data.

\section{Data Collection Procedure}

Consent was secured, and confidentiality was ensured. Nurses' participation in this research is entirely voluntary. The study used primary and secondary data collection procedures. The researchers looked for qualified informants. They prepared a semi-structured list of questions before the actual interview, including discussing the informant profile. Researchers asked follow-up questions and clarifications. The interview was taperecorded to ensure correct interpretation and analysis. Using voice recordings during the interview is vital since the researchers could gather the exact information an informant wants to tell.

Secondary data that were used in this study are the copy of the informants' PRC identification card and certificate of employment to prove that they are employed by their respective employees and are qualified in the inclusion criteria of the subject.

\section{Data Analysis}

After collecting the data, the researchers followed the steps to be undertaken in analyzing qualitative data. Cool and warm analyses were used. The whole process was based on the data, transcribed interviews, sorting, categorizing (cool analysis), categories as thematized (warm analysis). [17] This process led to discover the value of the lived experiences of Emergency Department (ED) Nurses with comorbidities amidst the COVID-19 pandemic. The researchers interviewed ten participants using a semi-structured type consisting of pre-determined questions. Moreover, the researchers allowed the participants to answer the questions based on their thoughts and feelings freely. Interviews were tape-recorded to ensure that the researchers could collect the exact information from the participants. The next step undertaken was the transcription, wherein the researchers transcribed the verbal and recorded answers of the informants and turned them into text form. The use of tape recorders will be helpful as they are not bounded by limited time in listening to what the informants have said, thus, making it easier for them to create a written copy of the latter's answers. After the transcription, the researchers did the coding. It required them to assign a code to extracted significant statements from the field text. They proceeded to cluster and categorization, in which they converted the extracted data into manageable units. Similar ideas were also grouped as one in this step. The last step is to form themes wherein major, minor, and subthemes emerge based on the informants' answers.

\section{Preliminary Biases}

An overwhelming sense of dread and mental burden was found during COVID times. Sharing experiences with colleagues showed a common theme of burnout due to multiple factors, including emotional influence brought about by patients from COVID-19 as well as anxiety, stress, staffing shortages, insufficient basic medical facilities, wearing gears for long hour shifts, the need to distance self from family, the mandatory swabbing, and the fear of getting infected with possible impending death. A review of the literature found that these factors had significant prevalence in nursing experience during COVID-19.

Because of the realization that this experience is physically and emotionally draining, what about those nurses who already have comorbidities yet were task to handle COVID-19 patients in a very psychologically stressful work setting like ED. Studies show that people suffering from health conditions such as respiratory or cardiac disease and diabetes, or conditions that affect their immunity, are considered at high risk of getting infected with COVID-19 and could develop a life-threatening 
situation.[18] This study has influenced the researchers to conceptualize this research.

Based on preliminary data, anticipated findings would show the predominance of multiple factors in a negative experience. Presumably, these factors could be more common in a hospital setting where the chance of getting COVID cases is higher, like ED, and it could be worse if the nurses involved are with comorbidities.

\section{RESULTS AND DISCUSSION}

The pandemic propelled health workers, even with comorbidities, to stay on the front lines to treat patients. Using Colaizzi's phenomenological method, the lived experiences of nurses with comorbidities as front liners during this pandemic are explored, described, and analyzed accordingly.
Based on the extracted responses from a semi-structured one-on-one interview with Covid-19 Nurse front liners, the following themes and sub-themes emerged:

\section{Perceptions on the Emergence of Covid-19 Pandemic}

This theme revolves around the nurses' thoughts with comorbidities as they face the threats of the Covid-19 pandemic. The participants of the study accounted for their perceptions which can be represented by psychological distress among individuals caused by the Covid-19 pandemic, health care sector as an essential part of society in times of pandemic, and Unpreparedness of Healthcare Institutions and Medical Front liners to respond on the Unprecedented Setbacks of Covid-19.

Table 1: Major Theme 1: Perceptions on the Emergence of Covid-19 Pandemic

\begin{tabular}{|c|c|c|}
\hline Sub-themes & $\begin{array}{l}\text { Meaningful } \\
\text { (Frequency) }\end{array}$ & Percentage \\
\hline Psychological distress among individuals caused by Covid-19 pandemic & 7 & $53.85 \%$ \\
\hline $\begin{array}{l}\text { The unpreparedness of healthcare institutions and medical front liners to respond to the } \\
\text { unprecedented setbacks of Covid-19. }\end{array}$ & 4 & $30.77 \%$ \\
\hline The health care sector is an essential part of society in times of pandemic & 2 & $15.38 \%$ \\
\hline Total & 13 & $100 \%$ \\
\hline
\end{tabular}

Table 1 presents the perception of the participants on the repercussions of the Covid-19 pandemic, in general. There are 13 meaningful units extracted from the interview related to this major theme. Of the subthemes, the highest percentage is on Psychological Distress Among Individuals Caused by Covid-19 Pandemic (53.85\%). Following this is the unpreparedness of Healthcare Institutions and Medical Front liners to Respond to the Unprecedented Setbacks of Covid-19 (15.38\%) and the Health care sector as an essential part of society in times of pandemic (15\%), respectively.

\section{A. Subtheme 1.1: Psychological Distress Among Individuals Caused by Covid-19 Pandemic}

Participants believe that covid-19 brought distress to people, especially to them as health care workers. The following accounts show fear and anxiety among individuals:

- COVID19 brought fears to many people -- one of which is entering hospitals. Instead of seeking for cure in the hospital, people prefer to stay at home and find alternative solutions. Aside from the fact that people do not like entering hospitals, healthcare providers are also isolated in the community since people believe that they are exposed to the virus. (N2T1C1-3)

- I got devastated, I blamed the government for letting in tourists in our country that carry the virus, and their actions took too long to take effect; it was late. (N4T1C1)

- It is scary, given that it is my first time to experience such a health crisis on a global scale. There is this feeling of 
uncertainty, fear, anxiety, and helplessness. (N6T1C1-2)

- I never thought that this would happen. I only see this in movies. I never expected that one day, a virus will change how we live, how we do things, how we deal with other people. This is a new experience. Though I am scared, I know we'll get through this health crisis. (N7T1C1)

- It is unprecedented, and it is scary. No one is certain what will happen to us. And because of uncertainty and fear, it brings confusion. (N9T1C1-2)

- The pandemic considerably changed our life. Everything became difficult. It paralyzed our community from functioning. It stopped people from doing their routines. (N10T1C1-3)

- Nurses are facing trials in this pandemic. They suffer from exhaustion and burnout because of highly demanding care and the increasing volume of patients having Covid 19. (N1T1C1-3)

Healthcare workers, especially nurses at the frontlines, pose a high risk of developing depression and anxiety due to significant pressures due to this Covid-19 pandemic. Nurses who encountered social rejection by the family had approximately three times the odds of being depressed. Those who felt rejected by the neighbors had five times odds due to their work related to caring for a COvid-19 patient. In addition, nurses who frequently watch the news associated with COVID-19 had approximately three times the odds of suffering from depression. [19]

Furthermore, those who belong to the female gender, have a sedentary lifestyle, smoker, have moderate and poor health conditions, those who regret their profession because of the pandemic and related unexpected experiences, who do not update themselves on the latest COVID-19related research, who experience discrimination in the workplace, and who face social problems due to working in a lab or hospital have a significantly high score in the hospital anxiety and depression scale. [20]

\section{B. Subtheme 1.2: Unpreparedness of Healthcare Institutions and Medical Front liners to respond to the Unprecedented Setbacks of Covid-19.}

Some responses reflect the unpreparedness of healthcare institutions and healthcare providers as they admit lacking as Covid-19 front liners.

- I know that we are not properly equipped with the resources to fight COVID-19. (N5T1C2-3)

- Our healthcare system is not ready for this scenario, and that makes us vulnerable, especially we are on the frontline in the battle against this war. (N6T1C3-4)

- Policies and procedures are from scratch; everything seems trial and error. The feeling is bizarre. As a healthcare worker, I also haven't experienced such a pandemic my entire career. I thought it's just only in history. No one saw this coming. (N9T1C2-5)

- I never thought that this would happen. I only see this in movies. I never expected that one day, a virus would change how we live, how we do things, how we deal with other people. This is a new experience. Though I am scared, I know we'll get through this health crisis. (N7T1C1)

Covid-19 brought anxiety to healthcare workers, especially nurses. [21] Due to low perceived preparedness to Covid-19, nurses are faced with psychological distress that affects their work performance as well as their physical and mental health. Shortage in staffing, as well as hospital beds and medical supplies, including personal protective equipment, are the major concerns that nurses are facing in this time of the pandemic. In addition, those healthcare workers reported increased stress and burnout are due to low perceived preparedness to respond to COVID-19. [22] 
Subtheme 1.3: Health care sector as an essential part of society in times of pandemic

The participants expressed that in this trying time, other people realize their worth as health care providers. The following narratives exemplify this:

- "It is only now that they appreciated nurses and other healthcare providers because of the pandemic." (N3T1C1)

- The pandemic greatly changed our life. Everything became difficult. It paralyzed our community from functioning. It stopped people from doing their routines. But, it also made the healthcare sector more of an important part of society. (N10T1C1-4)

There is no denying that nurses play a crucial role in mitigating this current health crisis. Nurses are predominantly depicted as the health care worker that provides direct patient care and they are well represented at every level to mitigate this pandemic starting from advising governments to being forefront in conducting research, management, and coordination of public health groups as well as in designing plans for humanitarian responses to COVID-19. [23] Nurses are the largest component of the health workforce, and they play a vital role in developing effective nursing practice and aid in preserving the core values of health systems on a global scale. Nurses are now presuming responsibilities in COVID19 interventions, and they will continue to be key players in mitigating this pandemic with adequate assistance. [24]

\section{Psychological State}

This theme revolves around the psychological state of the nurses with comorbidities as they face the challenges that this Covid-19 pandemic brings. The participants of the study mentioned their psychological, which can be represented by the occurrence of negative and positive behaviors when working in a high-risk workplace and a sense of professional responsibility.

Table 2: Major Theme 2: Psychological State

\begin{tabular}{|l|l|l|}
\hline Sub-themes & $\begin{array}{l}\text { Meaningful Units } \\
\text { (Frequency) }\end{array}$ & Percentages \\
\hline Occurrence of negative and positive behaviors when working in the high-risk workplace. & 13 & $72.2 \%$ \\
\hline Sense of professional responsibility & 5 & $27.8 \%$ \\
\hline Total & 18 & $100 \%$ \\
\hline
\end{tabular}

Table 2 shows the psychological state of the participants on the impact of the COVID-19 pandemic.

There are 18 meaningful units extracted from the interview related to this major theme. Of the subthemes, the highest percentage is on the occurrence of negative and positive behaviors when working in a high-risk workplace, followed by a sense of professional responsibility.

\section{A. Subtheme 2.1: Occurrence of negative and positive behaviors when working in a high-risk workplace}

While the majority of the participants expressed negativity about their current situation, some found a positive outlook on it. The following accounted for their mixed behaviors.
- I felt scared at first when this pandemic started. We didn't have the proper training to deal with this crisis, but then I stood as a health worker and faced the fear and just did the basic concepts in nursing (N1T2C7-9)

- I could not hide the fact that I was afraid of the virus. I mean, I am more fearful of the fact that my family could be infected because of me. That's why I am extra careful. (N2T2C10-12)

- I felt scared, especially that I must be extra cautious this time, especially that the symptoms of the COVID virus can be easily transferred from one person to another. (N3T2C3-5)

- It is a good thing that we strictly follow the protocols/guidelines in our hospital. 
Though, still, we must be extra careful. (N3T2C6-7)

- I was skeptical at first. What if I will acquire the virus? What if I could pass on my family? I was pessimistic then. I was frightened, really. (N4T2C7-9)

- I felt that this would be my final year it's because of the effect of the virus on many health care workers that most cases affected by the virus are dead. So with this case rate, I am scared. (N5T2C4-6)

- I am afraid of the said virus. I don't want to be infected, most especially my family. (N5T2C7-8)

- At first, I hesitated. I am worried that I might acquire the disease, might infect my family, and discriminate against us. I feared that I might die, and I thought about what will happen to my family. To be honest, I am afraid. I have hypertension, to begin with. I have learned that persons with comorbidities are more at risk of serious complications once they acquire covid-19. (N6T2C712)

- I am excited. This pandemic made me appreciate our profession more. We are helping people in need of our care. Also, I never tried wearing such PPE before. Though it was quite uncomfortable, the experience was new. It also made me hone my assessment and triaging skills as well. (N7T2C5-8)

- I feel anxious. I know what to do, but with this uncertainty, I do not know what to expect. The influx of patients might make me catch the disease. To begin with, I have diabetes, and that makes me a high-risk individual. I asked my seniors if they could spare me from having my duty at ER, but they said that my expertise could help the unit function well. In the end, those words of encouragement and their trust in my nursing skills overshadowed my anxiety and fear. (N7T2C9-14)

- I was very anxious, of course! Because you do not know where your enemy (virus) is coming from, is it at work, is the people you work with, is it the patient itself, or the watchers. It felt very uneasy and uncomfortable, but my sanity was still intact. (N8T2C10-12)

- I am a human too. I got afraid. I worry. I feel anxious; especially I have hypertension to think too. I think about my family. I thought of quitting because of my comorbidity, but I decided to work. I need to work not only for the people in need but also for the ones I love. (N9T2C11-14)

- I am so confused. I am full of doubts and uncertainties in my mind. It also reached a point that I also doubted myself. I battled myself in silence. I reflected on whether or not I should accept the duties that were given to me. (N10T2C9-12)

Although every job entails some risk for injury, risks vary widely across individuals, jobs, sectors, and workplaces. Studies have confirmed that learning ability, professional behavior, safety and accidents, and risk perception are affected by state anxiety and trait anxiety. Anxiety is associated with risk perception. In addition, the magnified risk may be discerned by individuals with high levels of anxiety and may probably lead to fear or withdrawal behaviors. [25].

\section{Subtheme 2.2: Sense of Professional Responsibility}

The participants faced their responsibility despite the risks of contracting the disease. Their answers were proof that they were ready to respond to the call of duty.

- I must accept my responsibilities and duties as a health care worker in our country to give the best prompt care for patients that are suffering. (N1T2C4-6)

- Well, I had to. It is a part of my duties and responsibilities as a nurse. It was indeed scary in the beginning, but now, I think, it is already "normal". (N2T2C79)

- Being a health care provider, it feels like there was no choice but to go on duty 
even on the verge of the pandemic. I just thought it was my kind of call of duty. (N4T2C4-6)

- I felt like I had no choice. I am a nurse, and whether I like it or not, it's my job; it's my profession. I was not prepared to be taking care of patients with covid-19, but nurses must be adaptive to change; they must be flexible. I believe that before myself, I should serve. I am not acting like a hero, but I am sworn to help people in need. (N9T2C6-10)

- It's a battle for all of us. And I believe that I have to do my part. I was given the task to be assigned to an area where few nurses would like to work in. I need to do my best. (N10T2C17-19)

Despite health risks for themselves and their loved ones, nurses are expected to provide care, and this may subject them to a greater degree of ethical pressure. [26] Health care professionals have continued to fulfill work-related and family-related responsibilities despite the pandemic. However, health care professional's fear of infecting their family members affects family relationships negatively, which makes them self-isolate. [27]

\section{Practiced Protective Behaviors}

This theme revolves around the way the participants practice precautionary measures to protect and prevent them from getting the disease along with their families. These behaviors were noted on two subthemes which consist of self-protective behaviors and precautionary measures set for the family.

Table 3: Major Theme 3: Practiced Protective Behaviors

\begin{tabular}{|l|l|l|}
\hline Sub-themes & $\begin{array}{l}\text { Meaningful Units } \\
\text { (Frequency) }\end{array}$ & Percentages \\
\hline $\begin{array}{l}\text { Self-Protective } \\
\text { Behaviors }\end{array}$ & 7 & $50 \%$ \\
\hline $\begin{array}{l}\text { Precautionary Measures } \\
\text { Set for Family }\end{array}$ & 7 & $50 \%$ \\
\hline Total & 14 & $100 \%$ \\
\hline
\end{tabular}

\section{A. Subtheme 3.1: Self-Protective Behaviors}

The participants were compliant in practicing self-protective behavior through the following activities.
- First of all, I took care of myself first. I make sure that before going home after my duty, I'll do the standard precaution. I changed my uniform at the hospital and taking a bath outside before entering our house. (N1T3C10-13)

- Before I go home, I make sure that I change my clothes in the hospital. I always bring with me alcohol. I disinfect my whole set of clothes before I enter our house. (N2T3C13-15)

- I eat nutritious foods, take supplements, have enough sleep and rest, practice minimum health standards such as performing hand washing and wearing a face mask, and at work with the use of PPE, and I also make sure to observe physical distancing. I make sure that I have alcohol with me when hand washing is not possible. (N6T3C13-17)

- As a nurse, I make sure to follow minimum health standards. I believe that prevention is better than cure. I cannot afford to acquire the disease both physically, emotionally, and financially. As much as possible, most of our expenses are spent on antibacterial soaps, alcohol, and vitamins. Also, I make sure to take a bath before entering the house after my duty. And I see to it that symptoms associated with Covid are well observed. (N7T3C15-20)

- I just followed health and safety protocols and taught my family as well. Giving myself time to pause and breathe. (N8T3C14-15)

- I take meticulous personal care to prevent myself from acquiring the disease. I practice minimum health standards. I wear PPE at work all the time. I wash my hands frequently than ever. I take a bath before I leave the hospital and another when I get home. I wash my uniforms first by soaking them in chlorine overnight before I wash them. (N9T3C15-19)

- I am religious when it comes to taking care of personal health. I cannot afford to become sick. I wash my hands, strengthen my immune system through 
foods and activities, and give myself adequate rest periods. (N10T3C20-22)

- Nurses' strategy in providing care for patients with COVID-19 has responsible self-protection. It is evident in the practice of delivering care in moderation, supplying own protective equipment, and strictly observing standard precautions. [28]

\section{B. Subtheme 3.2: Precautionary Measures Set for Family}

The participants make sure that their families are being protected during this time of pandemic by practicing the following measures.

- When in terms of their needs, I make sure that they have supplied with medication and the standard measures using mask and face shield, and I also educate them by not going to crowded places and what they must wear when going out. (N1T3C13-16)

- I am encouraging them to take their vitamins every day that is good for their immune system. I always advise them to stay at home as much as possible and go out only if there is a need to buy necessities in the market. I also buy them alcohol, face masks, and face shields. (N3T3C8-12)

- I reviewed all the necessary procedures. I see to it that the minimum safety protocols were followed. I wouldn't mingle with them after duty unless I got to take a bath and disinfect myself. (N4T3C10-12)

- Following the precautionary measures with my family \& educating them to do the right thing for us not to be infected by the virus (N5T3C9-10)

- As to my family, my responsibilities are still taken care of, I can still manage as long as I am well, and multitasking is in my vocabulary. (N8T3C15-17)

- As to my family, I always remind them to take vitamins and eat nutritious foods. I always told them not to go out if not necessary. But if they do, I always remind them to wear masks and face shields, and I always give them alcohol. (N9T3C19-22)

- My family and I wash our hands, strengthen our immune system through foods and activities, and give ourselves adequate rest periods. We always make sure to be informed of different knowledge about covid-19 to be able to fully protect ourselves, the family from acquiring it. (N10T3C22-24)

Nurses implement behavioral and environmental modifications to prevent the transmission of COVID-19. They also supply their family members with nutritious foods to increase their immunity and consistently remind them to consume supplements. In addition, nurses perform health education to improve their families' awareness of the importance of maintaining a healthy lifestyle. [29]

\section{Coping Mechanism}

This theme revolves around the ability of participants to find different strategies in coping with this battle. Their coping mechanisms can be categorized into two which are cognitive restructuring and self-care- physically, socially, mentally, spiritually, and emotionally.

Table 4: Major Theme 4. Coping Mechanism

\begin{tabular}{|l|l|l|}
\hline Sub-themes & $\begin{array}{l}\text { Meaningful Units } \\
\text { (Frequency) }\end{array}$ & Percentages \\
\hline Cognitive Restructuring & 4 & $28.6 \%$ \\
\hline $\begin{array}{l}\text { SELF-CARE (Physical, } \\
\text { Social, Mental, Spiritual } \\
\text { and Emotional Self-Care) }\end{array}$ & 10 & $71.4 \%$ \\
\hline Total & 14 & $100 \%$ \\
\hline
\end{tabular}

\section{A. Subtheme 4.1: Cognitive Restructuring}

Some of the participants can find and change inaccurate negative thoughts and be able to replace them with less stressinducing thoughts. The following are the manifestations of these habits.

- Though I am afraid, I always tell myself to be strong. To face my fears for myself, my family, and for everyone. (N10T4C16-17)

- We don't have the proper training to deal with this crisis, but then I stand as a health worker and face the fear and just 
do the basic concepts in nursing (N1T2C8-9)

- I feel anxious, and especially I have hypertension to think too. I think about my family. I thought of quitting because of my comorbidity, but I decided to work. I need to work not only for the people in need but also for the ones I love. (N9T2C11-14)

- ...those words of encouragement and their trust in my nursing skills overshadowed my anxiety and fear. (N7T2C13-14)

\section{B. Subtheme 4.2: SELF-CARE (Physical, Social, Mental, Spiritual and Emotional Self-Care)}

As they are devoted to helping others, most of them observe self-care to maintain good health holistically while dealing with the uncertainty of this pandemic. Their self-care behaviors are as follows.

- During duty off-days, I make sure that I do the things that I want to do, like visiting the seashore for some sort of relaxation. I also travel to places emphasizing the beauty of nature. (N2T4C16-18)

- Sleeping makes me feel relaxed. I spend a lot in my room doing the things I love to do. It lessens the stress and pressure that I feel whenever I am at the hospital. (N3T4C13-15)

- During my rest day, I spend most of my time sleeping because of the very exhausting type of work during this pandemic, and I have extra time I play basketball near our house just to reduce stressful situations during this crisis. (N5T4C11-14)

- I am fond of watching TV series during my off days, do planting in our backyard, take care of my kids, and play games on my phone. I make sure that I always have time for these things. (N6T4C20-22)

- Nurses cope with these feelings and emotions, one of which is thru meditation and deep breathing exercises to eliminate the negativity from within. (N1T4C17-19)

- I pray. I believe that my best weapon is God. Though I have hypertension, I make sure to ask for His guidance every time. I also do activities to lessen the stress due to the nature of my work. (N6T4C17-19)

- I pray a lot. I know that God is in control of everything. I meditate, I reflect, I try to see things positively. I read a bible verse; I listen to calming music. I make sure I think clearly. And I talk to people who have a positive outlook on life. (N7T4C21-24)

- A few of my strategies include talking to God (praying and listening to online Mass and Preaches), having chitchats with family and friends, and selfreflecting. (N8T4C18-19)

- I am a family guy. Whenever I am down, I always turn to my family for moral support. I also talk to God for guidance and blessing not only for myself and my family but also to all. I make sure to spend quality time with my family whenever possible. (N9T4C2326)

- Mental health is very important to me. That is why I make sure that I can give myself a breather when I am exhausted about work and life in general. I pray, do relaxation activities, do things that I love the most, and spend quality time with my family. This pandemic is indeed stressful, and I openly talk to my friends whenever I feel like depression is kicking me. Short talks about life always help me reset my mind. (N10T4C2530)

\section{CONCLUSION AND RECOMMENDATION}

As reflected from the result of this study, nurses who are at the frontline service and are currently working in hospitals have different interventions in providing healthcare services to COVID-19 patients. They are one of the key players in stopping the spread of the virus disease with adequate 
assistance from their respective institutions. Occupational safety is crucial during COVID-19 as health threats in their workplaces surround nurses. They must be protected during the pandemic. Further, nurse leaders must ensure that prevention and security interventions are carried out and applied to reduce workplace hazards, especially for health workers who have health problems. Lastly, hospitals must have appropriate and up-to-date infection control protocols and adequate personal protective equipment, especially for healthcare providers assigned to monitor and care for suspected or confirmed COVID-19 patients.

Therefore, researchers recommend that health care institutions having nurses with comorbidities must consider their health issues by not letting them be exposed to COVID 19 areas. In giving quality, safe, and efficient health care services, health care institutions must support their healthcare workers by sending them to various training and seminar workshops specifically on how to handle patients with COVID- 19 and learn more about how to properly use protective equipment, and get updated with the latest trends, ways, and procedures that can improve nursing services. Also, hospitals must strictly implement and adhere to the policies and procedures concerning fighting the COVID19 to minimize the risk of getting the said virus, especially to health workers with comorbidities.

Furthermore, hospital administrators must ensure that all medical supplies are available to help keep the lives of nurses and patients safe so that nurses must be able to perform their roles and responsibilities effectively. Finally, further studies such as the exploration of the experiences of nurses must be made, and additional studies on pandemic crises involving preparedness, response, and recovery and nurses' levels of knowledge, preparedness, and risk perception, which affects their adherence to precautionary behaviors must also be conducted as these concepts are critical points that must be addressed in this time of the pandemic.

\section{Declaration}

The authors received no financial support for this article's research, authorship, and publication. The authors have no conflict of interest to declare.

\section{Acknowledgement: None}

\section{Conflict of Interest: None}

\section{Source of Funding: None}

\section{REFERENCES}

1. Nasrin, G., Kamran, A., Toulabi, T., \& Heydari, H. Exploring nurses' experiences of psychological distress during care of patients with COVID-19: a qualitative study. BMC Psychiatry. 2020 Oct 6;20(1): 489. doi: 10.1186/s12888-020-02898-1.

2. Kelly, H. (2011). Bulletin of the World Health Organization.

3. Zhu H, Wei L, Niu P. (2020). The novel coronavirus outbreak in Wuhan, China.

4. Adalja. A., Toner, E. \& Inglesby, T. (2020). Priorities for the US health community responding to COVID-19.

5. Powers, R. \& Daily, E. (2010). International disaster nursing. Cambridge: Cambridge University Press.

6. Xie, J., Tong, Z., Guan, X., Du, B. \& Qiu, H., \& Slutsky, A. (2020). Critical care crisis and some recommendations during the COVID-19 epidemic in China. Intensive Care Med.

7. Al Thobaity, A.\&Alshammari, F. (2020). Nurses on the Frontline against the COVID19 Pandemic: An Integrative Review.

8. Healy, S. and Tyrrell, M. (2011). Stress in emergency departments: experiences of nurses and doctors: Sonya Healy and Mark Tyrrell review accounts of acute stress among healthcare professionals and show how it can be anticipated, reduced and managed.

9. Hooper, C., Craig, J., Janvrin, D., Wetsel, M., \& Reimels, E. (2010). Compassion satisfaction, burnout, and compassion fatigue among emergency nurses compared with nurses in other selected inpatient specialties. 
Mariecris Talosig et.al. Lived experiences of emergency department (ED) nurses with comorbidities amidst covid-19 pandemic at Candon City of Ilocos Sur: a phenomenological study.

10. Boyle, D. (2011). Countering Compassion Fatigue: A Requisite Nursing Agenda.

11. Liu M, Cheng SZ, Xu KW, Yang Y, Zhu QT, Zhang H, Yang DY, Cheng, SY, Xiao H, Wang JW, yao HR, Cong YT, Zhou YQ, Peng, S, Kuang M, Hou FF, Chweng KK, \& Xiao HP. Use of personal protective equipment against coronavirus disease 2019 by healthcare professionals in Wuhan, China: a cross-sectional study.

12. Xiang YT, Yang Y, Li W, Zhang Q, Cheung $\mathrm{T}, \mathrm{Ng} \mathrm{CH}$. Timely mental health care for the 2019 novel coronavirus outbreak is urgently needed. Psychiatry. 2020 Mar;7(3):228-229. doi: 10.1016/S2215-0366(20)30046-8. Epub 2020Feb 4. PMID: 32032543; PMCID: PMC7128153

13. Kolcaba's Comfort Theory |Nursology. (n.d.)

14. Christensen, C., Welch, A., \& Barr, J. (2017).Husserlian Descriptive Phenomenology: A review of intentionality, reduction, and the natural attitude.

15. Crossman, A. (2020). Understanding Purposive Sampling.

16. Gurven, M., Blackwell, A., Rodriguez, D., Stieglitz, J., and Kaplan, H. (2012). Does blood pressure inevitably rise with age?: longitudinal evidence among foragerhorticulturalists.

17. Moreno, A. \& Daño, J. (2018). From Dystopia to Utopia: The Perceptions of Ostomy Clients.

18. Ejaz, H., Alsrhani, A., Zafar, A., Javed, H., Junaid, K., Abdalla, A. E., sAbosalif, K. O. A., Ahmed, Z., \&Younas, S. (2020). COVID-19 and comorbidities: Deleterious impact on infected patients. In Journal of Infection and Public Health (Vol. 13, Issue 12, pp. 1833-1839). Elsevier Ltd.

19. Marthoenis, M., Fathiariani, L., \& Nassimbwa, J. (2021). Investigating the burden of mental distress among nurses at a provincial COVID-19 referral hospital in Indonesia: a cross-sectional study.

20. Tasnim, R. et al. (2021). Prevalence and correlates of anxiety and depression in frontline healthcare workers treating people with Covid-19 in Bangladesh.
21. Al Thobaity, A.\&Alshammari, F. (2020). Nurses on the Frontline against the COVID19 Pandemic: An Integrative Review.

22. Afulani, P., Gyamerah, A., Nutor, J., Laar, A., Aborigo, R., Malechi, H., Sterling, M., \& Awoonor-Williams, J. (2021). Inadequate preparedness for response to COVID-19 is associated with stress and burnout among healthcare workers in Ghana.

23. Schwerdtle, P. N., Connell, C., Lee, S., Plummer, V., Russo, P., Endacott, R., and Kuhn, L. (2020). Nurse Expertise: A Critical Resource in the Coivd-19 Pandemic Response.

24. Fawas, M., Anshasi, h., and Samaha, A. (2020). Nurses at the Front Line $f$ of COVID-19: Roles, Responsibilities, Risks, and Rights. 2021.

25. Liu, X., Hu, J., Xu, X., Xianyu, Y., and Dong, W. (2021). Factors influencing risk perception and nosocomial infection prevention practices of frontline nurses during the Covid-19 pandemic.

26. Sperling, D. (2020). Ethical Dilemmas, perceived risk, and motivation among nurses during the COVID-19 pandemic.

27. Ehrlich, H., McKenney, M., \& Elkbuli, A. (2020). Protecting our healthcare workers during the COVID-19 pandemic.

28. Moradi, Y., Baghaei, R., Hosseingholipour, K., \& Mollazadeh. F. (2021). Protective reactions of ICU nurses providing care for patients with COVID-19: a qualitative study.

29. Widiasih, R., Emaliyawati, E., Hedrawati, S., Susanti, R. D., Sutini, T., Sari, C. W. M. (2021). Nurses' Actions to Protect their families from Covid-19; A Descriptive Qualitative Study.

How to cite this article: Talosig M, Sanchez R, Gevin Soriano G. Lived experiences of emergency department (ED) nurses with comorbidities amidst COVID-19 pandemic at Candon City of Ilocos Sur: a phenomenological study. Int J Health Sci Res. 2021; 11(9): 216229. DOI: https://doi.org/10.52403/ijhsr. 20210934 